\title{
The Space-Jump Model of the Movement of Tumor Cells and Healthy Cells
}

\author{
Meng-Rong Li, ${ }^{1}$ Yu-Ju Lin, ${ }^{1}$ and Tzong-Hann Shieh ${ }^{2}$ \\ ${ }^{1}$ Department of Mathematical Sciences, National Chengchi University, No. 64, Section 2, ZhipNan Road, \\ Wenshan District, Taipei 11605, Taiwan \\ ${ }^{2}$ Department of Aerospace and Systems Engineering, Feng Chia University, No. 100 Wenhwa Road, Seatwen, \\ Taichung 40724, Taiwan
}

Correspondence should be addressed to Tzong-Hann Shieh; thshieh@fcu.edu.tw

Received 24 January 2014; Accepted 23 March 2014; Published 9 April 2014

Academic Editor: Malay Banerjee

Copyright (C) 2014 Meng-Rong Li et al. This is an open access article distributed under the Creative Commons Attribution License, which permits unrestricted use, distribution, and reproduction in any medium, provided the original work is properly cited.

We establish the interaction model of two cell populations following the concept of the random-walk, and assume the cell movement is constrained by space limitation primarily. Furthermore, we analyze the model to obtain the behavior of two cell populations as time is closed to initial state and far into the future.

\section{Introduction}

In the 1980s, the movement of isolated single cells was researched and was modelled by a range of authors (Oster [1]; Oster and Perelson [2]; Bottino and Fauci [3]; and Bottino, et al. [4]). In mathematics and biomedicine, not only of one-cell population but of multiple cell populations, there are many researches on the movement.

A consequential early paper written by Keller and Segel [5] modelled a partial differential equation to study the biochemical regulation of bacteria movement; their research has been the basis for models of the movement of diversified cell populations, such as slime mould aggregation (Höfer et al. [6]), tumor angiogenesis (Chaplain and Stuart [7]), primitive streak formation (Painter et al. [8]), and wound repair (Pettet et al. [9]).

In the recent years, most of the researches on cell movement focused on the interaction of multiple cell populations, precise cell behavior, and the development of the mathematics modelling. In this study we follow the contour of two-cell interaction developed by Painter and Sherratt [10]. The modelling of interaction of tumor- and healthycell populations was developed with the concept of randomwalk (space-jump). Assuming the movement is according to space limitation and the diffusion coefficients of two cell populations are the same, we develop a system of partial differential equations (PDEs). Through some calculations, the system of PDEs is simplified to a system of ordinary differential equations (o.d.es.). Analyzing the system of o.d.es., it is obtained that the number of two cell populations per unit area in a unit amount of time is finite no matter when; namely, the density of each cell population does not blow up.

To model the motion of biological organisms, there are three major concepts which would be used:

(a) the space-jump process in which the individual jumps between sites on a lattice,

(b) the velocity-jump process in which discontinuous changes in the speed or direction of an individual are generated by a Poisson process,

(c) the flux motion in which the movement of cells are treated as the flux motion.

In this work we adopt space-jump concept to establish our model and from it we show how a PDE of cell movement could be deduced. Then we use the same concept and expand the PDE which has been deduced to reason a system of PDEs describing the interaction of two cell population.

\section{Movement of One-Cell Population}

We will deduce an equation of cell movement on a lattice from the space-jump concept; moreover, we translate that equation 
into a PDE of cell movement through changing variables. First, we list the functions and variables that will be used in this content and call the considering cell population by $u$-cell as follows:

$u\left(x_{i}, t\right) \equiv u_{i}$ number of $u$-cell at site $x_{i}$ at time $t$ per unit area in a unit amount of time (the density of $u$ cell at site $x_{i}$ at time $t$ ),

$E\left(x_{i}, t\right) \equiv E_{i}$ the information of $u$-cell at site $x_{i}$ at time $t$,

$g\left(E_{i+1}\right)$ the probability of $u$-cell moving from $x_{i}$ to $x_{i+1}$ (to right),

$g\left(E_{i-1}\right)$ the probability of $u$-cell moving from $x_{i}$ to $x_{i-1}$ (to left).

Moreover, the meaning of $g\left(E_{i+1}\right)$ is that the probability of the cell moving to the target would be influenced by the information of the cell's jumping target.

For example, we choose that the cell density on position $x_{i+1}$ at time $t$ is the information of cells on $x_{i+1}$ at $t$; then the probability of cells moving from $x_{i}$ to $x_{i+1}$ would be influenced by $E_{i+1}$, which is the density of cell population on position $x_{i+1}$ at time $t$. Reasonably, a decreasing function $g\left(E_{i+1}\right)$ with respect to $E_{i+1}$ implies that a lower probability results from the more crowded target.

Supposing that cells move continuously in time on a lattice (discrete space), a PDE of $u$-cell movement would be modelled.

In the lattice space, the $u$-cells' movement at time $t$ can be modelled as

$$
\begin{aligned}
\frac{\partial u_{i}}{\partial t}= & g\left(E_{i}\right)\left(D_{u}\left(x_{i-1}, t\right) u\left(x_{i-1}, t\right)+D_{u}\left(x_{i+1}, t\right) u\left(x_{i+1}, t\right)\right) \\
& -D_{u}\left(x_{i}, t\right) u\left(x_{i}, t\right)\left(g\left(E_{i-1}\right)+g\left(E_{i+1}\right)\right) .
\end{aligned}
$$

We explain our idea as shown in Figure 1.

Figure 1 shows the movement of cells; the function on the figure is the moving probability. The changing of the $u$ cell density at site $x_{i}$ at time $t$ is equal to that of the $u$-cell number jumping from site $x_{i-1}$ and site $x_{i+1}$ minus the $u$ cell number jumping to site $x_{i-1}$ and site $x_{i+1}$. $\partial u_{i} / \partial t$ means the changing of $u$-cell density at site $x_{i}$ and time $t$. The function $g\left(E_{i}\right) D_{u}\left(x_{i-1}, t\right) u\left(x_{i-1}, t\right)+g\left(E_{i}\right) D_{u}\left(x_{i+1}, t\right) u\left(x_{i+1}, t\right)$ is the increase of $u$-cell density at site $x_{i}$ at time $t$ with cells moving from site $x_{i-1}$ and site $x_{i+1}$ to site $x_{i}$, where $D_{u}\left(x_{i}, t\right)$ is the jumping (diffusion) coefficient of $u$-cell at site $x_{i}$ at time $t$. And $-D_{u}\left(x_{i}, t\right) u\left(x_{i}, t\right)\left(g\left(E_{i-1}\right)+g\left(E_{i+1}\right)\right)$ is the decrease of $u$-cell density at site $x_{i}$ at time $t$ with cells moving to site $x_{i-1}$ and site $x_{i+1}$ from site $x_{i}$. Thus, (1) is obtained.

The model of $u$-cell movement in continuous space can be deduce from (1) in a lattice space through changing variables. Let $x_{i+k}=x+k h, k \in \mathbb{Z} . x_{i}=x, x_{i+1}=x+h, x_{i-1}=x-h$, and $E_{i}=E(x, t)$; hence, (1) becomes

$$
\begin{aligned}
\frac{\partial u(x, t)}{\partial t}=g(E(x, t)) & \left(D_{u}(x-h, t) u(x-h, t)\right. \\
& \left.+D_{u}(x+h, t) u(x+h, t)\right)
\end{aligned}
$$

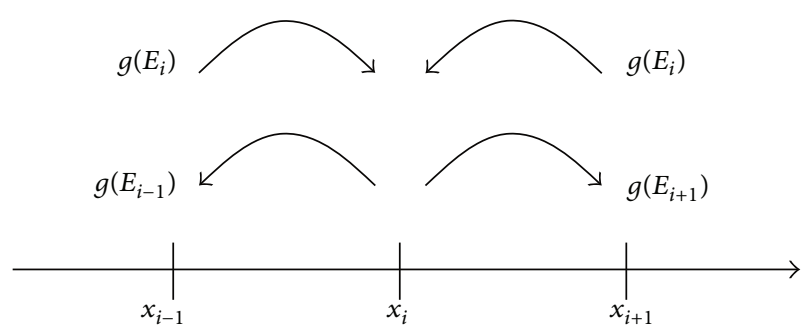

FIGURE 1: The movement of cells.

$$
\begin{aligned}
& -(g(E(x-h, t))+g(E(x+h, t)) \\
& \left.\quad \times D_{u}(x, t) u(x, t)\right) .
\end{aligned}
$$

For a continuum flow we consider that the jumping coefficient $D_{u}(x, t)=D_{u}$ is a constant. Denote $u(x-h, t)$ and $u(x+h, t)$ by Taylor's series

$$
\begin{aligned}
u(x-h, t)= & u(x, t)+\frac{\partial u}{\partial x}(x-h-x) \\
& +\frac{1}{2 !} \frac{\partial^{2} u}{\partial x^{2}}(x-h-x)^{2}+\cdots \\
u(x+h, t)= & u(x, t)+\frac{\partial u}{\partial x}(x+h-x) \\
& +\frac{1}{2 !} \frac{\partial^{2} u}{\partial x^{2}}(x+h-x)^{2}+\cdots
\end{aligned}
$$

In consequence, $u(x-h, t)+u(x+h, t)=2 u(x, t)+$ $\left(\partial^{2} u / \partial x^{2}\right) h^{2}+O\left(h^{4}\right) ;$ similarly,

$$
\begin{aligned}
g(E(x-h, t))+g(E(x+h, t))= & 2 g(E(x, t)) \\
& +\frac{\partial^{2} g}{\partial x^{2}} h^{2}+O\left(h^{4}\right) .
\end{aligned}
$$

Consequently,

$$
\begin{aligned}
\frac{\partial u}{\partial t}(x, t)= & g(E) D_{u} \frac{\partial^{2} u}{\partial x^{2}} h^{2}+O\left(h^{4}\right) g(E) \\
& -D_{u} u \frac{\partial^{2} g}{\partial x^{2}} h^{2}-D_{u} u O\left(h^{4}\right),
\end{aligned}
$$

and then we get

$$
\frac{\partial u}{\partial t}=D_{u} \frac{\partial}{\partial x}\left(g(E) \frac{\partial u}{\partial x}-u \frac{\partial g(E)}{\partial x}\right) h^{2}+O\left(h^{4}\right) .
$$

Therefore, we consider (1) as the following.

The $u$-cell movement can be modelled as

$$
\frac{\partial u(x, t)}{\partial t}=D_{u} \frac{\partial}{\partial x}\left(g(E) \frac{\partial u}{\partial x}-u \frac{\partial g(E)}{\partial x}\right),
$$

where $D_{u}$ is a diffusion coefficient and $E(x, t) \equiv E$ is the information of $u$-cell on position $x$ at time $t$. 


\section{Interaction of Two Cell Populations}

Now we show how to deduce a system of PDEs which describes the interaction of two cell populations. Here the two considered cell populations are called by $u$-cell and $v$-cell. What the variables and functions $(E(x, t)$ and $g(E))$ mean is as above; moreover, denote the density of $u$-cell and $v$ cell populations on position $x$ at time $t$ by $u(x, t)$ and $v(x, t)$, respectively. On the other hand, we write $w(x, t):=u(x, t)+$ $v(x, t)$ to describe the total cell density. There is also another vague function, $g(E)$, which needs to be defined clearly.

Given that space limitation influences the movement of cells, the probability of cells moving to position $x$ decreases with how the position is crowded with cells. We choose $w(x, t)$, the total cell density, to express the information of cells on position $x$, namely, $E(x, t)=w(x, t)$. Hence $g(E)=$ $g(w)=1-(w / T)$ shows that the probability of cells moving to position $x$ decreases with the total cell density on position $x$, where $T \gg w$ initially and $T$ is a constant. Here the assumption on $g(E)$ follows the paper written by Painter and Sherratt (2003) [10].

After defining those variables, the model of interaction of two cell populations ( $u$-cell and $v$-cell) can be deduced. According to $(7)$, replacing $g(E)$ by $1-(w(x, t) / T) \equiv 1-$ $(w / T)$, then

$$
\begin{aligned}
\frac{\partial u}{\partial t} & =D_{u} \frac{\partial}{\partial x}\left(\left(1-\frac{w}{T}\right) \frac{\partial u}{\partial x}+u \frac{\partial}{\partial x}\left(1-\frac{w}{T}\right)\right) \\
& =D_{u} \frac{\partial}{\partial x}\left(\frac{\partial u}{\partial x}-\frac{w}{T} \frac{\partial u}{\partial x}+\frac{u}{T} \frac{\partial w}{\partial x}\right) \\
& =D_{u} \frac{\partial}{\partial x}\left(\frac{\partial u}{\partial x}-\frac{v}{T} \frac{\partial u}{\partial x}+\frac{u}{T} \frac{\partial v}{\partial x}\right) \\
& =D_{u}\left(\frac{\partial^{2} u}{\partial x^{2}}-\frac{v}{T} \frac{\partial^{2} u}{\partial x^{2}}+\frac{u}{T} \frac{\partial^{2} v}{\partial x^{2}}\right),
\end{aligned}
$$

where $D_{u}$ is a constant. Similarly, the same processes are applied to $v$. We obtain the following equation:

$$
\frac{\partial v}{\partial t}=D_{v}\left(\frac{\partial^{2} v}{\partial x^{2}}-\frac{u}{T} \frac{\partial^{2} v}{\partial x^{2}}+\frac{v}{T} \frac{\partial^{2} u}{\partial x^{2}}\right)
$$

Consequently, we get the interaction of two cell populations.

Following space limitation, the interaction of two cell populations can be modelled as

$$
\begin{aligned}
& \frac{\partial u}{\partial t}=D_{u}\left(\left(1-\frac{v}{T}\right) \frac{\partial^{2} u}{\partial x^{2}}+\frac{u}{T} \frac{\partial^{2} v}{\partial x^{2}}\right), \\
& \frac{\partial v}{\partial t}=D_{v}\left(\left(1-\frac{u}{T}\right) \frac{\partial^{2} v}{\partial x^{2}}+\frac{v}{T} \frac{\partial^{2} u}{\partial x^{2}}\right),
\end{aligned}
$$

where $D_{u}$ and $D_{v}$ are diffusion coefficients with respect to $u$ cell and $v$-cell $\left(D_{u}\right.$ and $D_{v}$ are constants), respectively.

Furthermore, through changing variables,

$$
\mu \equiv \mu(x, t)=\frac{u(x, t)}{T}, \quad v \equiv v(x, t)=\frac{v(x, t)}{T},
$$

with the consequence that

$$
\frac{\partial u}{\partial t}=T \frac{\partial \mu}{\partial t}, \quad \frac{\partial v}{\partial t}=T \frac{\partial v}{\partial t}, \quad \frac{\partial^{2} u}{\partial x^{2}}=T \frac{\partial^{2} \mu}{\partial x^{2}}, \quad \frac{\partial^{2} v}{\partial x^{2}}=T \frac{\partial^{2} v}{\partial x^{2}}
$$

Rewriting system (10) as

$$
\begin{aligned}
& T \frac{\partial \mu}{\partial t}=D_{\mu}\left((1-v) \frac{\partial^{2} \mu}{\partial x^{2}}+\mu \frac{\partial^{2} v}{\partial x^{2}}\right) T, \\
& T \frac{\partial \nu}{\partial t}=D_{\nu}\left((1-\mu) \frac{\partial^{2} v}{\partial x^{2}}+\nu \frac{\partial^{2} \mu}{\partial x^{2}}\right) T,
\end{aligned}
$$

the system of P.D.Es (10) can be simplified as

$$
\begin{aligned}
& \frac{\partial \mu}{\partial t}=D_{\mu}\left((1-v) \frac{\partial^{2} \mu}{\partial x^{2}}+\mu \frac{\partial^{2} v}{\partial x^{2}}\right), \\
& \frac{\partial \nu}{\partial t}=D_{\nu}\left((1-\mu) \frac{\partial^{2} v}{\partial x^{2}}+\nu \frac{\partial^{2} \mu}{\partial x^{2}}\right),
\end{aligned}
$$

where $D_{\mu}$ and $D_{\nu}$ are diffusion coefficients.

Now, the interaction of $u$-cell and $v$-cell has been modelled. Model (14) will be used frequently in the following context, and some properties of two cell populations can be deduced from analyzing model (14). We show the analyzing procedures and some results in the next section.

\section{The Behavior and the Meaning of $\nu(x, t)=v(z)$ as $z \rightarrow 0$}

We have got the system of PDEs (14) which shows the interaction of two cell populations. In this section, model (14) will be transformed to a system of o.d.es. and then analyzed to obtain some properties of $\nu(x, t)=v(z)$ as $z$ approaches to zero and infinite; furthermore, the properties of $\mu(x, t)=$ $\mu(z)$ will be deduced from the properties of $\nu(z)$ and $\omega(z)$, where $\omega(z)$ is $\mu(z)+\nu(z)$.

Our purpose is to obtain a simpler form of (14) in order to analyze the model conveniently. Supposing that $u$ cell and $v$-cell have the same diffusion coefficient $\left(D_{\mu}\right.$ is equal to $\left.D_{\nu}\right), k$ denotes the diffusion coefficients $D_{\mu}$ and $D_{\nu}$. Through changing variables, the system of PDEs (14) could be transformed to a system of o.d.es.

Lemma 1. Given two cell populations with the same diffusion coefficient, the system of PDEs (14) can be shown as a system of o.d.es. as follows:

$$
\begin{aligned}
& -\frac{1}{2} z \mu^{\prime}(z)=k\left((1-v) \mu^{\prime \prime}(z)+\mu \nu^{\prime \prime}(z)\right), \\
& -\frac{1}{2} z \nu^{\prime}(z)=k\left((1-\mu) \nu^{\prime \prime}(z)+v \mu^{\prime \prime}(z)\right),
\end{aligned}
$$

where $z=x / \sqrt{t}, k \equiv D_{\mu}=D_{v}$. 
Proof. According to the system of PDEs (14), we could obtain

$$
\begin{aligned}
& \frac{\partial \mu}{\partial t}=k\left((1-v) \frac{\partial^{2} \mu}{\partial x^{2}}+\mu \frac{\partial^{2} v}{\partial x^{2}}\right), \\
& \frac{\partial \nu}{\partial t}=k\left((1-\mu) \frac{\partial^{2} v}{\partial x^{2}}+\nu \frac{\partial^{2} \mu}{\partial x^{2}}\right) .
\end{aligned}
$$

Let $\mu(z)=\mu(x / \sqrt{t}) \equiv \mu(x, t)$ and $\nu(z)=\nu(x / \sqrt{t}) \equiv$ $\nu(x, t)$, with the consequence that

$$
\begin{aligned}
& \frac{\partial \mu(x, t)}{\partial t} \equiv-\frac{1}{2} x t^{-3 / 2} \mu^{\prime}\left(\frac{x}{\sqrt{t}}\right), \\
& \frac{\partial \nu(x, t)}{\partial t} \equiv-\frac{1}{2} x t^{-3 / 2} \nu^{\prime}\left(\frac{x}{\sqrt{t}}\right), \\
& \frac{\partial^{2} \mu(x, t)}{\partial x^{2}} \equiv t^{-1} \mu^{\prime \prime}\left(\frac{x}{\sqrt{t}}\right), \\
& \frac{\partial^{2} \nu(x, t)}{\partial x^{2}} \equiv t^{-1} \nu^{\prime \prime}\left(\frac{x}{\sqrt{t}}\right) .
\end{aligned}
$$

The system of PDEs (16) can be written as model (15).

In that case, the simpler form (model (15)) will be analyzed in the following subsections in order to obtain some properties of $\nu(z)$.

Before deducing that $v(x, t)=v(z)$ is bounded for $z$ in $[0, \delta]$ ( $\delta$ is very small), we must know the behavior of total cells.

Lemma 2. The movement of total cells (u-cell and v-cell) can be modelled as a classical diffusion equation $\omega^{\prime \prime}(z)+$ $(z / 2 k) \omega^{\prime}(z)=0$.

Proof. Adding the two equations in the system (15), we obtain

$$
\mu^{\prime \prime}(z)+\frac{z}{2 k} \mu^{\prime}(z)+\nu^{\prime \prime}(z)+\frac{z}{2 k} \nu^{\prime}(z)=0 .
$$

Imposing $\omega(z)$ upon (18), equation (18) could be rewritten as follows:

$$
\omega^{\prime \prime}(z)+\frac{z}{2 k} \omega^{\prime}(z)=0
$$

In consequence,

$$
\omega(z)=\omega\left(z_{0}\right)+\omega^{\prime}\left(z_{0}\right) \int_{z_{0}}^{z} e^{-r^{2} / 4 k} d r
$$

where $z_{0}=x_{0} / \sqrt{t_{0}}$, for some site $x_{0}$ at initial time $t_{0}$.

According to above assumptions, $\omega(x, t) \equiv \omega(z)=\mu(z)+$ $\nu(z)$ and $\mu(z)=u(z) / T$ and $\nu(z)=v(z) / T, \omega(z)$ can be restored to $(u(z) / T)+(v(z) / T)$, where $T$ is a constant. In that case, equation (20) can be transformed into the form

$$
\left(\frac{u+v}{T}\right)(z)=\left(\frac{u+v}{T}\right)\left(z_{0}\right)+\left(\frac{u+v}{T}\right)^{\prime}(z) \int_{z_{0}}^{z} e^{-r^{2} / 4 k} d r
$$

and then written as

$$
(u+v)(z)=(u+v)\left(z_{0}\right)+(u+v)^{\prime}\left(z_{0}\right) \int_{z_{0}}^{z} e^{-r^{2} / 4 k} d r,
$$

where $z$ is $x / \sqrt{t}$ and $k$ is a constant. The last equation shows the behavior of total cells; moreover, that is the classical representation of the solution of the fundamental diffusion equation.

After describing the behavior of total cells, following (15), we replace $\mu$ by $\omega-v$ in the equation

$$
-\frac{1}{2} z \mu^{\prime}(z)=k\left((1-\nu) \mu^{\prime \prime}(z)+\mu \nu^{\prime \prime}(z)\right) .
$$

Hence,

$$
-\frac{1}{2} z(\omega-v)^{\prime}(z)=k\left((1-v)(\omega-v)^{\prime \prime}(z)+(\omega-v) \nu^{\prime \prime}(z)\right) \text {. }
$$
fied as

Given that $-(z / 2 k) \omega^{\prime}=\omega^{\prime \prime}$, the equation (24) is simpli-

$$
(\omega(z)-1) \nu^{\prime \prime}(z)-\frac{z}{2 k} \nu^{\prime}(z)-\omega^{\prime \prime}(z) \nu(z)=0,
$$

where $\omega(z)$ is as (20), with the consequence that

$$
\nu^{\prime \prime}(z)+\frac{(-z)}{2 k(\omega(z)-1)} v^{\prime}(z)+\frac{\left(-\omega^{\prime \prime}(z)\right)}{\omega(z)-1} \nu(z)=0 .
$$

Lemma 3. Equation (26) can be transformed to

$$
\bar{\nu}^{\prime \prime}(z)+a(z) \bar{v}(z)=0,
$$

where

$$
a(z)=\frac{1+2 \omega^{\prime}\left(z_{0}\right) z e^{-z^{2} / 4 k}}{4 k(\omega(z)-1)}-\frac{4 k \omega^{\prime}\left(z_{0}\right) z e^{-z^{2} / 4 k}+z^{2}}{16 k^{2}(\omega(z)-1)^{2}} .
$$

Proof. Assuming that $\nu(z)=\bar{\nu}(z) \exp \left((1 / 2) \int^{z} p(r) d r\right)$, equation (26) is transformed as follows:

$$
\bar{\nu}^{\prime \prime}(z)+\left(q(z)-\frac{1}{2} p^{\prime}(z)-\frac{1}{4} p^{2}(z)\right) \bar{\nu}(z)=0,
$$

where $p(z)=-z / 2 k(\omega(z)-1)$ and $q(z)=-\omega^{\prime \prime}(z) /(\omega(z)-1)$. Hence we denote $a(z)$ as $q(z)-(1 / 2) p^{\prime}(z)-(1 / 4) p^{2}(z)$.

Therefore,

$$
\begin{aligned}
a(z)= & \frac{-\omega^{\prime \prime}(z)}{\omega(z)-1}-\frac{1}{2} \frac{-2 k(\omega(z)-1)+z 2 k \omega^{\prime}(z)}{4 k^{2}(\omega(z)-1)^{2}} \\
& -\frac{1}{4} \frac{(-z)^{2}}{4 k^{2}(\omega(z)-1)^{2}} \\
= & \frac{\omega^{\prime}\left(z_{0}\right) z e^{-z^{2} / 4 k}}{2 k(\omega(z)-1)} \\
& +\frac{4 k(\omega(z)-1)-4 k \omega^{\prime}\left(z_{0}\right) z e^{-z^{2} / 4 k}-z^{2}}{16 k^{2}(\omega(z)-1)^{2}} .
\end{aligned}
$$

Hence, $\bar{\nu}^{\prime \prime}(z)+a(z) \bar{v}(z)=0$, where $v(z)=$ $\bar{\nu}(z) e^{(1 / 2) \int^{z} p(r) d r}$. 
In order to simplify the representation of the following equations, we let

$$
\begin{aligned}
& a_{1}(z)=\frac{\omega^{\prime}\left(z_{0}\right) z e^{-z^{2} / 4 k}}{2 k(\omega(z)-1)}, \\
& a_{2}(z)=\frac{1}{4 k(\omega(z)-1)}, \\
& a_{3}(z)=-\frac{\omega^{\prime}\left(z_{0}\right) z e^{-z^{2} / 4 k}}{4 k(\omega(z)-1)^{2}}, \\
& a_{4}(z)=-\frac{z^{2}}{16 k^{2}(\omega(z)-1)^{2}} .
\end{aligned}
$$

The following theorem would show that $\bar{\nu}(z)$ and $v(z)$ are bounded on $[0, \delta]$ for some small $\delta$.

Before we make the following theorem complete, the substantiation of the next lemma must be finished.

Theorem 4. The solution of $\bar{\nu}^{\prime \prime}(z)+\left(-M_{0}^{2}+b(z)\right) \bar{v}(z)=0$ is bounded where $M_{0}$ is a constant and $b(z)$ is closed to zero as $z \ll 1$ if the solution of $\bar{\nu}^{\prime \prime}(z)+\left(-M_{0}^{2}\right) \bar{\nu}(z)=0$ is bounded as $z \ll 1$.

Proof. Assume $z \ll 1$; the solution of $\bar{\nu}^{\prime \prime}(z)+\left(-M_{0}^{2}\right) \bar{v}(z)=0$ is given by

$$
\bar{\nu}(z)=c_{1} e^{M_{0} z}+c_{2} e^{-M_{0} z},
$$

where $c_{1}$ and $c_{2}$ are constants.

We say that $\bar{\nu}_{1}(z)$ is the solution of $\bar{\nu}^{\prime \prime}(z)+\left(-M_{0}^{2}\right) \bar{\nu}(z)=0$ and $\bar{v}_{2}(z)$ is the solution of $\bar{\nu}^{\prime \prime}(z)+\left(-M_{0}^{2}+b(z)\right) \bar{v}(z)=0$. Then we have

$$
\begin{aligned}
\left|\bar{\nu}_{1}\right| & =\left|c_{1} e^{M_{0} z}+c_{2} e^{-M_{0} z}\right| \\
& \leq\left|c_{1}\right| e^{M_{0} z}+\left|c_{2}\right| e^{-M_{0} z} \\
& \leq\left|c_{1}\right| e^{M_{0} \delta}+\left|c_{2}\right|, \quad \forall z \in[0, \delta], \delta<1 .
\end{aligned}
$$

Let $\bar{v}_{21}(z)=\bar{\nu}_{2}(z), \bar{\nu}_{22}(z)=\bar{\nu}_{2}^{\prime}(z)$, and

$$
\begin{aligned}
& \bar{V}(z)=\left[\begin{array}{l}
\bar{\nu}_{21}(z) \\
\bar{v}_{22}(z)
\end{array}\right], \quad A=\left[\begin{array}{cc}
0 & 1 \\
M_{0}^{2} & 0
\end{array}\right], \\
& B(z)=\left[\begin{array}{cc}
0 & 0 \\
-b(z) & 0
\end{array}\right] .
\end{aligned}
$$
as

The equation $\bar{\nu}^{\prime \prime}(z)+\left(-M_{0}^{2}+b(z)\right) \bar{\nu}(z)=0$ can be written

$$
\frac{d}{d z} \bar{V}(z)=A \bar{V}(z)+B(z) \bar{V}
$$

Let $\Phi(z)$ be a fundamental solution matrix of $\Phi^{\prime}(z)=$ $A \Phi(z)$. Then

$$
\begin{aligned}
\bar{V}= & \Phi(z) \Phi^{-1}\left(z_{0}\right) \bar{V}\left(z_{0}\right) \\
& +\Phi(z) \int_{z_{0}}^{z} \Phi^{-1}(r) B(r) \bar{V}(r) d r
\end{aligned}
$$

$$
\begin{aligned}
\|\bar{V}\| \leq & \left\|\Phi(z) \Phi^{-1}\left(z_{0}\right) \bar{V}\left(z_{0}\right)\right\| \\
& +\int_{z_{0}}^{z}\left\|\Phi\left(z-r+z_{0}\right) \Phi^{-1}\left(z_{0}\right) B(r) \bar{V}(r)\right\| d r \\
\leq & M_{1} M_{2}+\int_{z_{0}}^{z} M_{1}\|B(r)\|\|\bar{V}\| d r,
\end{aligned}
$$

where $\|\cdot\|$ is the super norm and $M_{1}=\left\|\Phi(z) \Phi^{-1}\left(z_{0}\right)\right\|, M_{2}=$ $\left\|\bar{V}\left(z_{0}\right)\right\|$.

By Granwall's inequality and $\int_{z_{0}}^{z} M_{1}\|B(r)\| d r \quad \leq$ $M_{1}\|B(z)\| \delta$ for all $z$ in $[0, \delta]$, then

$$
\begin{aligned}
\|\bar{V}\| & \leq M_{1} M_{2} \exp \left(\int_{z_{0}}^{z} M_{1}\|B(r)\| d r\right) \\
& \leq M_{1} M_{2} \exp \left(M_{1}\|B(z)\| \delta\right)<\infty,
\end{aligned}
$$

for all $z$ in $[0, \delta]$.

Hence, the solution of $\bar{\nu}^{\prime \prime}(z)+\left(-M_{0}^{2}+b(z)\right) \bar{v}(z)=0$ is bounded as $z \ll 1$.

Theorem 5. $\bar{v}(z)$ is bounded on $[0, \delta]$ for some small $\delta$; moreover, $\nu(z)$ is bounded on $[0, \delta]$.

Proof. Supposing that $\omega(z)=\omega\left(z_{0}\right)+\omega^{\prime}\left(z_{0}\right) \int_{z_{0}}^{z} e^{-r^{2} / 4 k} d r$ is closed to $\omega\left(z_{0}\right)$ as $z \rightarrow 0^{+}$and $\omega\left(z_{0}\right)<1$, then $\omega(z)-1<0$ when $z \rightarrow 0^{+}$.

According to the above assumptions, we have

$$
\begin{aligned}
& a_{1}(z)=\frac{\omega^{\prime}(z) z e^{-z^{2} / 4 k}}{2 k(\omega(z))-1} \sim 0 \quad \text { as } z \sim 0, \\
& a_{2}(z)=\frac{1}{4 k(\omega(z)-1)} \sim-M_{0}^{2} \quad \text { as } z \sim 0, \\
& a_{3}(z)=\frac{-\omega^{\prime}\left(z_{0}\right) z e^{-z^{2} / 4 k}}{4 k(\omega(z)-1)^{2}} \sim 0 \quad \text { as } z \sim 0, \\
& a_{4}(z)=\frac{-z^{2}}{16 k^{2}(\omega(z)-1)^{2}} \sim 0 \quad \text { as } z \sim 0 .
\end{aligned}
$$

For $z \ll 1, a_{1}(z)+a_{3}(z)+a_{4}(z)=b(z), a(z)=-M_{0}^{2}+b(z)$ can be estimated immediately.

Thus the equation $\bar{\nu}^{\prime \prime}(z)+a(z) \bar{\nu}(z)=0$ can be written as

$$
\bar{\nu}^{\prime \prime}(z)+\left(-M_{0}^{2}+b(z)\right) \bar{\nu}(z)=0
$$

for all $z \ll 1$.

Because the solution of $\bar{\nu}^{\prime \prime}(z)+\left(-M_{0}^{2}\right) \bar{\nu}(z)=0$ is bounded as $z \rightarrow 0$, the solution of $\bar{\nu}^{\prime \prime}(z)+\left(-M_{0}^{2}+b(z)\right) \bar{v}(z)=0$ is also bounded as $z \rightarrow 0$. Consequently, $\bar{\nu}(z)$, the solution of $\bar{v}^{\prime \prime}(z)+\left(-M_{0}^{2}+b(z)\right) \bar{\nu}(z)=0$ for all $z \ll 1$, is bounded on $[0, \delta]$ for some small $\delta$, saying that $|\bar{\nu}(z)| \leq M$ and $M$ is a constant. 
Hence,

$$
\begin{aligned}
\nu(z) & =\bar{v}(z) \exp \left(\frac{-1}{2} \int^{z} p(r) d r\right) \\
& \leq M \exp \left(\frac{-1}{2} \int^{z} p(r) d r\right),
\end{aligned}
$$

where $p(z)=(-z) /(2 k(\omega(z)-1))>0$ for some $k>0$; moreover, since $p(z)>0, e^{((-1) / 2) \int^{z} p(r) d r} \leq 1$ for all $z$ in $[0, \delta]$ and for some $k>0$. In consequence, $v(z)$ is bounded by $M \exp \left((-1 / 2) \int^{z} p(r) d r\right)$ where $M$ is a constant and $p(z)=$ $-z / 2 k(\omega(z)-1)$ on $[0, \delta]$ for some $k>0$.

It is verified that $v(z)$ is bounded by $M \exp \left((-1 / 2) \int^{z} p(r) d r\right)$ where $M$ is a constant and $p(z)=-z / 2 k(\omega(z)-1)$ on $[0, \delta]$, where $z$ is $x / \sqrt{t}$ and $\delta$ is very small. Furthermore, we restore $v(z)$ to $v(x / \sqrt{t}) / T$, where $T$ is a positive constant. $z \rightarrow 0$ expresses that time $t$ approximates infinite. Therefore, Theorem 5 indicates that the density of $v$-cell population approximates finite number as time approaches infinite. Through writing $u(x / \sqrt{t})$ as $w(x / \sqrt{t})-v(x / \sqrt{t})$, it could be deduced immediately that the density of $u$-cell population is finite no matter how long time passes.

\section{The Behavior and the Meaning of}

$$
\nu(x, t)=v(z) \text { as } z \rightarrow \infty
$$

Near $z=0$ (namely, $x / \sqrt{t}$ approaches zero), the boundedness of $v(z)$ has been shown. Hence, we obtain that the density of $u$-cell and $v$-cell populations would not blow up when time approached infinity. In this section, through justifying that $\bar{v}(z)$ is bounded by $e^{z^{2} / 8 k \delta}$ first, we will show that $\nu(z)$ is also bounded when $z$ approaches $\infty$.

Theorem 6. The solution of $\bar{\nu}^{\prime \prime}(z)+a(z) \bar{v}(z)=0$, got by Lemma 3, is bounded by $e^{z^{2} / 8 k \delta}$ as $z$ approaches $\infty$, where $\delta>0$.

Proof. Supposing $\omega(z)=\omega\left(z_{0}\right)+\omega^{\prime}\left(z_{0}\right) \int^{z} \exp \left(-r^{2} / 4 k\right) d r$ approaches $1^{-}$, there is a $\delta>0$ such that $\omega-1$ approaches $-\delta$ as $z \rightarrow \infty$. As $z$ tends to infinity, $a(z)$ could be rewritten as the following asymptotic form:

$$
\begin{aligned}
a(z) & =\frac{2 \omega^{\prime}\left(z_{0}\right) z e^{-z^{2} / 4 k}+1}{4 k(\omega(z)-1)}-\frac{4 k \omega^{\prime}\left(z_{0}\right) z e^{-z^{2} / 4 k}+z^{2}}{16 k^{2}(\omega(z)-1)^{2}} \\
& \sim \frac{-1}{4 k \delta}-\left(\frac{z}{4 k \delta}\right)^{2}, \quad \text { as } z \longrightarrow \infty .
\end{aligned}
$$

Consider

$$
\bar{\nu}^{\prime \prime}(z)+\left(\frac{-1}{4 k \delta}-\left(\frac{z}{4 k \delta}\right)^{2}\right) \bar{v}(z)=0
$$

and let $\bar{\nu}_{1}(z)=e^{f(z)}$ be a solution of (43). Immediately,

$$
f^{\prime \prime}(z)+\left(f^{\prime}(z)\right)^{2}=\frac{1}{4 k \delta}+\left(\frac{z}{4 k \delta}\right)^{2}
$$

is obtained. Assume $f(z)=b_{0} z^{2}+b_{1} z+b_{2}$, where $b_{0}, b_{1}$, and $b_{2}$ are constants; then

$$
4 b_{0}^{2} z^{2}+4 b_{0} b_{1} z+b_{1}^{2}+2 b_{0}=\frac{1}{4 k \delta}+\left(\frac{1}{4 k \delta}\right)^{2} z^{2} .
$$

Consequently, $b_{0}=1 / 8 k \delta$ and $b_{1}=0$; then $f(z)=$ $\left(z^{2} / 8 k \delta\right)+b_{2}$. Hence, we get $\bar{\nu}_{1}(z)=b e^{z^{2} / 8 k \delta}$, where $b \in \mathbb{R}$.

Now let $\bar{\nu}_{2}$ be another solution of (43). Assume that $\bar{\nu}_{2}=$ $g(z) e^{z^{2} / 8 k \delta}, g^{\prime \prime}(z)+(z / 2 k \delta) g^{\prime}(z)=0$, with the consequence that $g(z)=g\left(z_{0}\right)+g^{\prime}\left(z_{0}\right) \int_{z_{0}}^{z} e^{\left(-r^{2}\right) / 4 k \delta} d r$. We get

$$
\bar{\nu}_{2}(z)=g\left(z_{0}\right) e^{z^{2} / 8 k \delta}+g^{\prime}\left(z_{0}\right) \int_{z_{0}}^{z} e^{\left(\left(z^{2} / 8 k \delta\right)+\left(-r^{2} / 4 k \delta\right)\right)} d r
$$

Moreover, $\int^{z} e^{\left(\left(z^{2} / 8 k \delta\right)+\left(-r^{2} / 4 k \delta\right)\right)} d r$ is convergent since $\left(z^{2} / 8 k \delta\right)+\left(-r^{2} / 4 k \delta\right)=\left(z^{2}-2 r^{2}\right) / 8 k \delta<0$, as $r>z / \sqrt{2}$. Therefore, the solution of $\bar{\nu}^{\prime \prime}(z)+a(z) \bar{v}(z)=0$ is

$$
\begin{aligned}
b e^{z^{2} / 8 k \delta}+\left(g\left(z_{0}\right) \exp \left(\frac{z^{2}}{8 k \delta}\right)+g^{\prime}\left(z_{0}\right)\right. \\
\left.\times \int_{z_{0}}^{z} \exp \left(\frac{z^{2}}{8 k \delta}+\frac{-r^{2}}{4 k \delta}\right) d r\right),
\end{aligned}
$$

and then

$$
\bar{\nu}(z) \leq\left(b+g\left(z_{0}\right)\right) e^{z^{2} / 8 k \delta}+M,
$$

where $b$ is a constant and $M$ is defined as $g^{\prime}\left(z_{0}\right)\left(\int_{z_{0}}^{z} \exp \left(\left(z^{2} / 8 k \delta\right)+\left(-r^{2} / 4 k \delta\right)\right) d r\right)$.

After substantiating that $\bar{\nu}(z)$ is bounded by $e^{z^{2} / 8 k \delta}$ as $z$ approaches $\infty$, where $\delta>0$, it is not difficult to verify that $\nu(z)$ is also bounded as $z$ approaches $\infty$.

Theorem 7. $v(z)$ is bounded when $z$ approaches $\infty$.

Proof. Given $z \gg 1$, in above Theorem 6, we have transformed

$$
\nu^{\prime \prime}(z)+\frac{(-z)}{2 k(\omega(z)-1)} v^{\prime}(z)+\frac{\left(-\omega^{\prime \prime}(z)\right)}{\omega(z)-1} \nu(z)=0
$$

to $\bar{v}^{\prime \prime}(z)+a(z) \bar{v}(z)=0$ through changing $v(z)$ to $\bar{\nu}(z) e^{(-1 / 2) \int^{z}(-z / 2 k(\omega(z)-1)) d r}$, and

$$
\begin{aligned}
& \bar{\nu}(z) \exp \left(\frac{-1}{2} \int^{z} \frac{-z}{2 k(\omega(z)-1)} d r\right) \\
& \quad \leq\left(\left(b+g\left(z_{0}\right)\right) e^{z^{2} / 8 k \delta}+M\right) \exp \left(\frac{-1}{2} \int^{z} \frac{r}{2 k \delta} d r\right) .
\end{aligned}
$$

In consequence,

$$
\begin{aligned}
v(z) & \leq\left(\left(b+g\left(z_{0}\right)\right) e^{z^{2} / 8 k \delta}+M\right) e^{-z^{2} / 8 k \delta} \\
& =\left(b+g\left(z_{0}\right)\right)+M e^{-z^{2} / 8 k \delta},
\end{aligned}
$$


where $b \in \mathbb{R}$ and $M \equiv g^{\prime}\left(z_{0}\right)\left(\int^{z} e^{\left(\left(z^{2} / 8 k \delta\right)+\left(-r^{2} / 4 k \delta\right)\right)} d r\right)$. Hence, $v(z)$ is bounded by

$$
\left(b+g\left(z_{0}\right)\right)+M e^{-z^{2} / 8 k \delta}
$$

as $z \rightarrow \infty$.

Restoring $z$ to $x / \sqrt{t}$, according to Theorem 7 , we know that $\nu(x / \sqrt{t})$ is bounded by $\left(b+g\left(z_{0}\right)\right)+M \exp \left(-x^{2} /(8 k \delta t)\right)$ as $x / \sqrt{t}$ approaches $\infty$; namely, $t$ approaches initial time. In consequence, it is obtained immediately that the density of $v$-cell population which is denoted by $v(x / \sqrt{t}) / T$ tends to a finite number as $v$-cell population has begun moving for a fleeting time. Furthermore, the density of $u$-cell population would also approximate a finite number for the same time.

If it is possible, we hope the solutions of (10) could be obtained by using our methods that were analytically used in $[11-18]$ or numerically used in $[19,20]$.

\section{Conflict of Interests}

The authors declare that there is no conflict of interests regarding the publication of this paper.

\section{Acknowledgment}

Thanks are due to Professor Long-Yi Tsai, Professor Tai-Ping Liu, Professor Ton Yang, and Professor Shih-Shien Yu for their continuous encouragement and discussions over this work, to Metta Education, Grand Hall, and Auria Solarfor for their financial assistance, and to the referee for his interest and helpful comments on this paper.

\section{References}

[1] G. F. Oster, "On the crawling of cells," Journal of Embryology and Experimental Morphology, vol. 83, pp. 329-364, 1984.

[2] G. F. Oster and A. S. Perelson, "Cell spreading and motility: a model lamellipod," Journal of Mathematical Biology, vol. 21, no. 3, pp. 383-388, 1985.

[3] D. C. Bottino and L. J. Fauci, "A computational model of ameboid deformation and locomotion," European Biophysics Journal, vol. 27, no. 5, pp. 532-539, 1998.

[4] D. Bottino, A. Mogilner, T. Roberts, M. Stewart, and G. Oster, "How nematode sperm crawl," Journal of Cell Science, vol. 115, no. 2, pp. 367-384, 2002.

[5] E. F. Keller and L. A. Segel, "Initiation of slime mold aggregation viewed as an instability," Journal of Theoretical Biology, vol. 26, no. 3, pp. 399-415, 1970.

[6] T. Höfer, J. A. Sherratt, and P. K. Maini, "Dyctyostelium discoideum: cellular self-organisation in an excitable biological medium," Proceedings of the Royal Society of London B, vol. 259, no. 1356, pp. 249-257, 1995.

[7] M. A. J. Chaplain and A. M. Stuart, "A model mechanism for the chemotactic response of endothelial cells to tumour angiogenesis factor," IMA Journal of Mathematics Applied in Medicine and Biology, vol. 10, no. 3, pp. 149-168, 1993.

[8] K. J. Painter, P. K. Maini, and H. G. Othmer, "A chemotactic model for the advance and retreat of the primitive streak in avian development," Bulletin of Mathematical Biology, vol. 62, no. 3, pp. 501-525, 2000.

[9] G. J. Pettet, H. M. Byrne, D. L. S. Mcelwain, and J. Norbury, "A model of wound-healing angiogenesis in soft tissue," Mathematical Biosciences, vol. 136, no. 1, pp. 35-63, 1996.

[10] K. J. Painter and J. A. Sherratt, "Modelling the movement of interacting cell populations," Journal of Theoretical Biology, vol. 225, no. 3, pp. 327-339, 2003.

[11] R. Duan, M.-R. Li, and T. Yang, "Propagation of singularities in the solutions to the Boltzmann equation near equilibrium," Mathematical Models and Methods in Applied Sciences, vol. 18, no. 7, pp. 1093-1114, 2008.

[12] M.-R. Li and Y.-L. Chang, "On a particular Emden-Fowler equation with non-positive energy $u^{\prime \prime}-u^{3}=0$ : mathematical model of enterprise competitiveness and performance," Applied Mathematics Letters, vol. 20, no. 9, pp. 1011-1015, 2007.

[13] M.-R. Li, "Estimates for the life-span of the solutions for some semilinear wave equations," Communications on Pure and Applied Analysis, vol. 7, no. 2, pp. 417-432, 2008.

[14] M.-R. Li, "Blow-up solutions to the nonlinear second order differential equation $u^{\prime \prime}=u^{p}\left(c_{1}+c_{2} u^{\prime}(t)^{q}\right)$," Taiwanese Journal of Mathematics, vol. 12, no. 3, pp. 599-622, 2008.

[15] M.-R. Li and J.-T. Pai, "Quenching problem in some semilinear wave equations," Acta Mathematica Scientia, vol. 28, no. 3, pp. 523-529, 2008.

[16] M.-R. Li, "On the Emden-Fowler equation $u(t) u^{\prime \prime}(t)=c_{1}+$ $c_{2} u^{\prime}(t)^{2}$," Acta Mathematica Scientia B, vol. 30 , no. 4 , pp. $1227-$ 1234, 2010.

[17] M.-R. Li, Y.-J. Lin, and T.-H. Shieh, "The flux model of the movement of tumor cells and healthy cells using a system of nonlinear heat equations," Journal of Computational Biology, vol. 18, no. 12, pp. 1831-1839, 2011.

[18] T.-H. Shieh, T.-M. Liou, M.-R. Li, C.-H. Liu, and W.-J. Wu, "Analysis on numerical results for stage separation with different exhaust holes," International Communications in Heat and Mass Transfer, vol. 36, no. 4, pp. 342-345, 2009.

[19] T.-H. Shieh and M.-R. Li, "Numeric treatment of contact discontinuity with multi-gases," Journal of Computational and Applied Mathematics, vol. 230, no. 2, pp. 656-673, 2009.

[20] M.-R. Li, Y.-T. Li, T.-H. Shieh, C. J. Yue, and P. Lee, "Parabola method in ordinary differential equation," Taiwanese Journal of Mathematics, vol. 15, no. 4, pp. 1841-1857, 2011. 


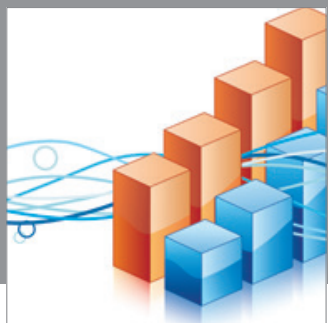

Advances in

Operations Research

mansans

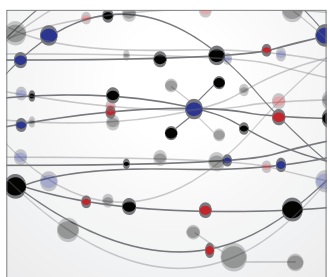

The Scientific World Journal
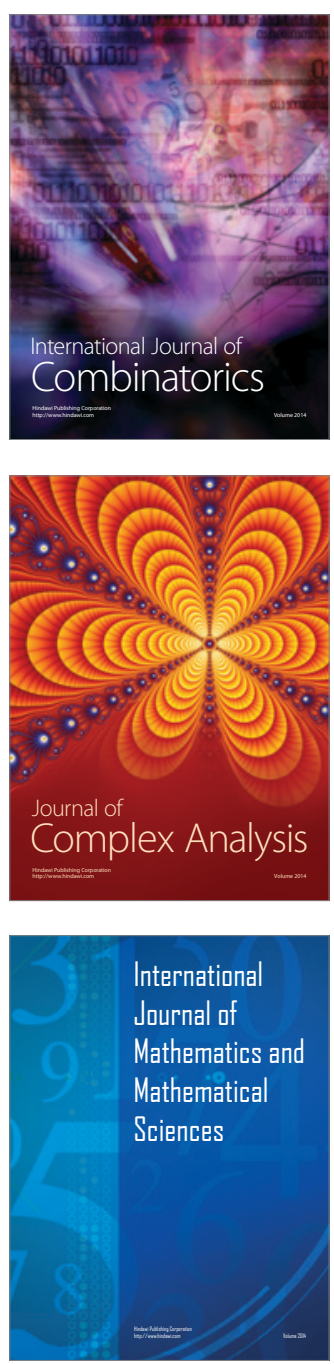
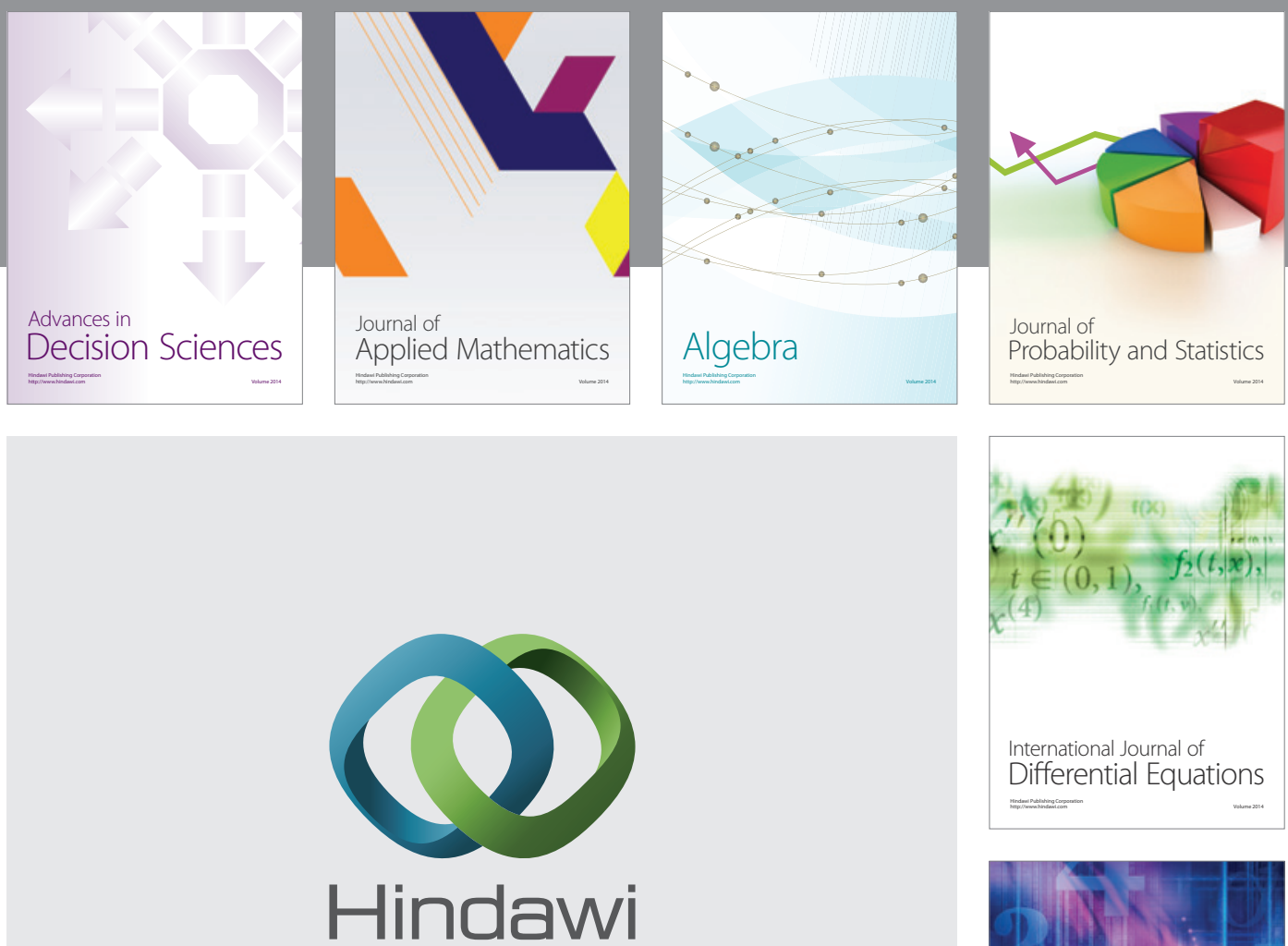

Submit your manuscripts at http://www.hindawi.com
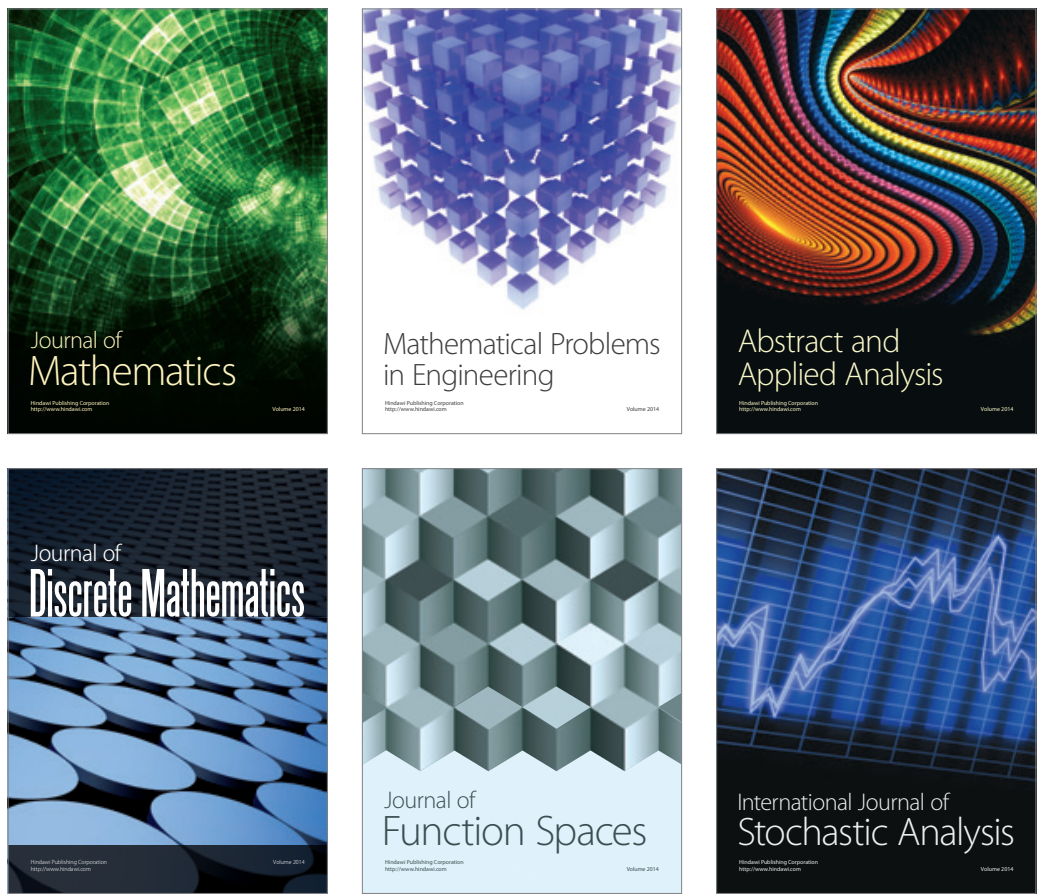

Journal of

Function Spaces

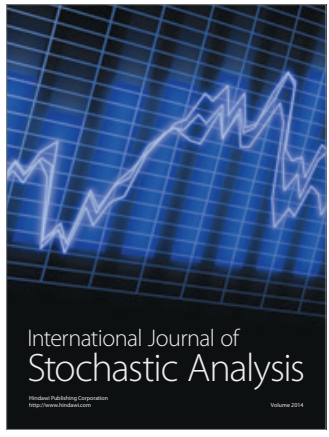

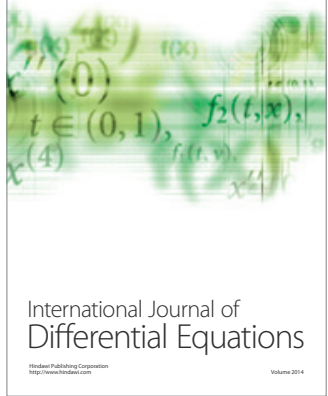
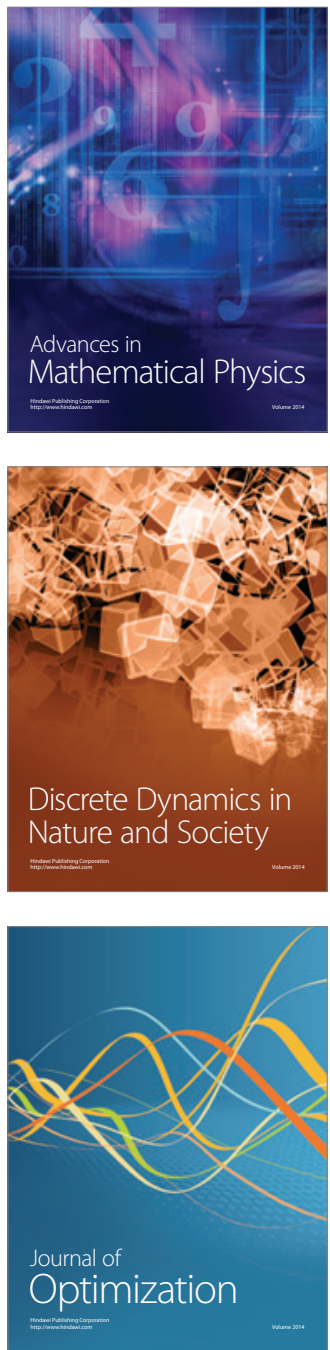